\title{
Identification of a Chimeric M4 Protein and Novel Emm Pattern in Currently Circulating Strains of Emm4 Group A Streptococcus
}

\section{Sruti DebRoy ${ }^{1}$, Xiqi Li ${ }^{1}$, Awdhesh Kalia ${ }^{3}$, Jessica Galloway Peña ${ }^{1,2}$ Brittany J. Shah $^{4}$, Vance G. Fowler, Jr. ${ }^{5}$, Anthony R. Flores ${ }^{4,6}$ and Samuel A. Shelburne $e^{1,2,5 \#}$}

${ }^{1}$ Department of Infectious Diseases Infection Control and Employee Health, ${ }^{2}$ Department of Genomic Medicine, ${ }^{3}$ Graduate Program in Diagnostic Genetics, School of Health Professions, University of Texas MD Anderson Cancer Center, Houston TX USA; ${ }^{4}$ Division of Infectious Diseases, Duke University Medical Center, Durham, NC,

${ }^{4}$ Division of Infectious Diseases, Department of Pediatrics, ${ }^{6}$ Center for Antimicrobial Resistance and Microbial Genomics, University of Texas Health Science Center McGovern Medical School, Houston, TX, USA.

*Corresponding author: Samuel A. Shelburne, MD, PhD, Department of Infectious Diseases, MD Anderson Cancer Center, Unit 1460, 1515 Holcombe Blvd, Houston, TX, 77030, 713-7923629 (o), 713-792-5381 (fax), sshelburne@mdanderson.org 


\begin{abstract}
Group A Streptococcus (GAS) is classified by sequence of the gene encoding the M protein (emm) and the patterns into which emm types are grouped. We discovered a novel emm pattern in emm4 GAS, historically considered pattern E, arising from a fusion event between emm and the adjacent enn gene. We identified the emm/enn fusion event in 51/52 emm4 GAS strains isolated by national surveillance in 2015. GAS isolates with an emm/enn fusion event completely replaced pattern E emm4 strains over a 4-year span in Houston (2013-2017). The novel emm/enn gene fusion and new emm pattern has potential vaccine implications.
\end{abstract}

Key words: group A Streptococcus; emm/enn gene fusion event; chimeric M protein; emm pattern 


\section{INTRODUCTION}

Group A Streptococcus (GAS) is among the most ubiquitous human pathogens and is classified into emm types based on the 5' sequence of the emm gene that encodes the hypervariable M protein, a key GAS virulence determinant [1]. GAS infection is thought to engender serotype-specific immunity, and the $\mathrm{M}$ protein or its domains are leading vaccine candidates [2]. In addition to invariably containing the emm gene, the $\mathrm{emm}$ region can also contain one or two emm-like genes which are typically designated as mrp and enn (Figure 1A) [3]. The 3' end of the emm and emm-like genes encode a peptidoglycan-spanning (PG) domain, and PG sequence variation determines four different subfamilies (SF-1 through SF-4, Figure 1A). The composition of emm-family genes along with their respective PG domain subfamily types divides GAS strains into five emm patterns (A-E), and for the overwhelming majority of GAS strains studied to date, there is strict concordance between emm type and emm pattern [3]. The emm pattern of GAS strains strongly correlates with the preferred epithelial site of infection i.e. pharynx versus skin [3]. Thus, emm pattern A-C strains are considered "throat specialists", whereas pattern D are "skin specialists”, and pattern E are "generalists” [4].

The majority of data establishing the five emm patterns and their relationship to $\mathrm{emm}$ type were generated from strains isolated in the 1980s and 1990s using targeted approaches such as PCR and Southern blot $[4,5]$. Initial whole genome sequencing (WGS) studies of one or a few GAS strains per emm type done in the 2000s have supported these findings [6]. However, the past decade has witnessed a marked increase in WGS of large cohorts of GAS strains which has revealed significant intra-emm type diversity [7]. Moreover, the highly antigenic and thus hypervariable nature of the $\mathrm{M}$ protein has long been recognized [8]. Therefore, with the 
additional density of sequencing data and the elapsed time since the recognition of the five $\mathrm{emm}$ patterns, one might predict that additional variations in the $\mathrm{emm}$ region would be recognized. Herein, we report the identification of genetic events in currently circulating emm4 strains, which were previously considered pattern E, that give rise to a chimeric M protein and a novel GAS emm pattern. 


\section{MATERIALS AND METHODS}

The invasive GAS emm4 strain SSGAS001 was isolated from a point-source outbreak in 2015 and its genome described using the strain name "Duke small” [9]. We accessed the complete genomes of the 2001 pharyngeal isolate MGAS10750, considered the emm4 reference strains, and the 2015 pharyngeal emm4 isolate MEW427 from NCBI [6, 10]. Short-read sequencing data of invasive emm4 strains collected by the Centers for Disease Control and Prevention (CDC) during 2015 [11] were accessed from BioProject PRJNA395240 on the shortread archive. Emm region sequences were extracted from annotated draft genomes and visualized through Geneious (Biomatters Ltd, NJ, USA). Two core single nucleotide polymorphism (SNP)-based methods were deployed to reconstruct WGS-based phylogenies: a) kSNP v3.0 identified core SNPs based on reference free k-mer analysis (k-mer size of 19, default settings). These SNPs were used to reconstruct a maximum parsimony tree; and b) parsnp v1.2 aligned genomes to the reference, identified SNPs in local collinear blocks, and subsequently reconstructed an approximate maximum-likelihood tree using FastTree 2. Due to similar findings, only trees reconstructed by parsnp are provided. 88 emm4 GAS were identified among a total of 930 sterile and non-sterile GAS isolates collected at Texas Children's Hospital in Houston, TX between 2013 and 2017 under a protocol approved by the Institutional Review Board at Baylor College of Medicine. The emm region was amplified by PCR and subject to Sanger sequencing (primer sequences are in Supplemental Table 1) with subsequent identification of the emm/enn gene fusion. 


\section{RESULTS}

Emm4 strains commonly cause both invasive and non-invasive GAS disease and are considered a prototype emm pattern E GAS [1, 12]. We previously compared the complete genome of an invasive emm4 strain isolated in 2015 (strain SSGAS001) to that of the reference emm4 strain MGAS10750, which was isolated in 2001 [6, 9]. Closer examination revealed a fusion event between emm and the immediately downstream enn gene in SSGAS001 resulting in a chimeric

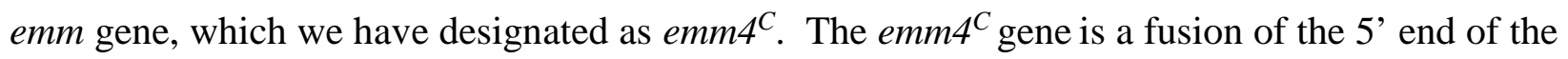
emm and the 3' end of the enn gene of MGAS10750 (Figure 1A). Given that the $5^{\prime}$ of emm4 ${ }^{C}$ is unchanged, SSGAS001 is still categorized as emm4. However, the C-terminal half of the emm4 $4^{C_{-}}$ encoded protein, $\mathrm{M} 4^{\mathrm{C}}$, only shares $62 \%$ identity with the C-terminus of the MGAS10750 M4 protein. Moreover, the chimeric M4 ${ }^{\mathrm{C}}$ protein now contains an SF-3 allele in the PG domain rather than the SF-2 allele found in the PG domain of the MGAS10750 M protein. In addition to generating a new chimeric M protein, the emm/enn gene fusion event observed in SSGAS001 also results in just two genes in the emm region ( $\mathrm{mrp}$ and emm) instead of the three ( $\mathrm{mrp}$, emm and enn) that are characteristic of Pattern E GAS (Figure 1A). Thus, the gene fusion event gives rise to an emm pattern heretofore undescribed in GAS strains. We analyzed the other fully sequenced emm4 GAS strain, MEW427, also isolated in 2015 [10], and found it to have the identical emm4 ${ }^{C}$ gene and emm pattern as strain SSGAS001.

Next, we wanted to determine the frequency of the gene fusion event amongst currently circulating emm4 GAS strains. We first analyzed published WGS data from 52 invasive emm4 isolates collected by the CDC in 2015 [11]. We identified the emm/enn gene fusion event in 51/52 strains with only a single strain containing emm pattern E (Figure 1B, Supplemental Table 2). 
Whole genome-based phylogenomics revealed that strains with the emm/enn gene fusion clustered distinctly from strains with the canonical emm4 gene (Figure 1B). We identified six variations of the $M 4^{\mathrm{C}}$ protein (Figure 2A, Supplemental Figure 1). The 350 amino acid long variant, which we have named $\mathrm{M} 4^{\mathrm{C}-350}$, occurred most commonly and was identical to $\mathrm{M}^{\mathrm{C}}$ in strains SSGAS001 and MEW427. M4 ${ }^{\mathrm{C}-350}$ differs from the other common M4 ${ }^{\mathrm{C}}$ variant, $\mathrm{M} 4^{\mathrm{C}-392}$, by a 126 nucleotide (42 amino acid) deletion (Figure $2 \mathrm{~A}$ ). Together $\mathrm{M} 4^{\mathrm{C}-350}$ and $\mathrm{M} 4^{\mathrm{C}-392}$ are present in $90 \%$ of strains in the CDC dataset that have the emm/enn gene fusion event (Figure 2B). The other four variants appear to be derived via insertions or deletions in the $\mathrm{M} 4^{\mathrm{C}-392}$ or $\mathrm{M} 4^{\mathrm{C}-350}$ protein (Supplemental Figure 1).

Although the $\mathrm{N}$-terminal of the $\mathrm{M} 4^{\mathrm{C}}$ proteins are identical to the canonical M4 protein, the $\mathrm{M} 4^{\mathrm{C}}$ proteins have different C-terminal repeat regions (CRRs). The CRRs represent a domain within the cell surface-exposed portion of the $\mathrm{M}$ and Enn proteins that contain tandemly arranged blocks of direct sequence repeats which have been targeted as potential GAS vaccine candidates [13]. The sequence repeats are not always identical and can be further classified based on the sequence of the C-terminal 14 amino acids (J14) in a given repeat [14]. The MGAS10750 M4 protein contains two CRRs, both of which have the J14.2 sequence. Unlike MGAS10750, M4-392 contains three CRRs, but all other 4 $^{\mathrm{C}}$ variants also possess two CRRs each. However, the composition of the CRRs in every $\mathrm{M}^{\mathrm{C}}$ variant differs from that in MGAS10750 in terms of the J14 sequence (Figure 2A, Supplemental Table 2, Supplemental Figure 1). Importantly, the M4 ${ }^{\mathrm{C}}$ proteins are present in strains isolated from multiple locations in the United States rather than being confined to a single location (Supplemental Figure 2).

To begin to assess temporal emergence of emm4 strains with the chimeric M protein, we analyzed 88 emm4 isolates collected at the Texas Children’s Hospital in Houston between 2013 
and 2017 via Sanger sequencing of the emm region (Supplemental Table 3). We identified the emm/enn gene fusion in 74 strains (84\%). While $66 \%$ of the emm4 strains isolated in 2013 were still the canonical emm pattern E, there was total replacement by strains carrying emm4 ${ }^{C}$ by 2017 (Figure 2C). Similar to the CDC strains, the $\mathrm{M} 4^{\mathrm{C}-350}$ and $\mathrm{M} 4^{\mathrm{C}-392}$ variants were most common among the Houston isolates. Consistent with the hypothesis that $\mathrm{M} 4^{\mathrm{C}-350}$ arose from $\mathrm{M} 4^{\mathrm{C}-392}, \mathrm{M} 4^{\mathrm{C}-}$ ${ }^{350}$-containing strains were not detected until one year after the initial identification of strains with the $\mathrm{M} 4^{\mathrm{C}-392}$ protein (Figure 2C). 


\section{DISCUSSION}

Since its discovery some 90 years ago, M protein has been considered the key GAS virulence determinant and is currently a prime vaccine candidate [2]. Despite extensive investigations over the past decades, continued study of $\mathrm{M}$ protein and the emm region continues to uncover new findings. Herein, we demonstrate that $>90 \%$ of recently circulating emm4 GAS strains contain a non-canonical chimeric M4 protein and a previously unrecognized emm pattern.

The key finding of this study was identification of a gene fusion event that gave rise to three novel entities. First, it created a chimeric emm gene, emm4 ${ }^{C}$, that consists of the 5 ' end of the reference emm4 gene and the 3' end of the enn4 gene. Second, formation of the chimeric emm $4^{C}$ gene eliminates the enn gene thereby producing an emm region that only contains $m r p$ and emm. Finally, unlike previously reported M proteins which harbor either the SF-1 or SF-2 allele in the PG domain [3], M4 ${ }^{\mathrm{C}}$ carries the SF-3 allele of the PG subfamily. To our knowledge, our study is the first report of a fusion event between emm family genes that alters the M protein as well as the first record of an emm pattern different from the five established ones.

Analysis of 140 recent emm4 isolates revealed that strains with the chimeric emm $4^{C}$ gene and the new emm pattern have almost completely replaced the previously circulating pattern $\mathrm{E}$ strains. This finding implies that the gene fusion event confers an advantage to the strains with the novel emm pattern or that strains with the gene fusion event have additional changes driving their proliferation. Given the critical nature of M protein in GAS host-pathogen interaction, it is plausible that the gene fusion event is at least a partial catalyst for the observed replacement. Multiple key aspects of GAS pathogenesis have been ascribed to M protein, such as binding of host molecules and inhibiting phagocytosis, although the precise function differs depending on M protein composition [1]. Additionally, M protein is the dominant GAS target of the human 
immune system [2]. Thus, the theoretical advantage conferred by $\mathrm{M}^{\mathrm{C}}$ protein could be due to augmented function of the chimeric $\mathrm{M}$ protein and/or to an improved ability to evade the host immune system.

In terms of altered susceptibility to human immunity, $\mathrm{M}$ protein sequence variation can confer changes in immune recognition and has been associated with clonal population expansion [7]. However, reports of M protein variation have described small-scale changes (i.e. insertions, deletions, tandem repeat variation) in the $\mathrm{N}$-terminal region whereas we observed replacement of the entire C-terminus $[7,8]$. Although the $\mathrm{N}$-terminus is typically considered to be the portion of the $\mathrm{M}$ protein under selective immune pressure [1], there are also data supporting immunogenicity of the C-terminal region. Specifically, J14 sequences in the CRRs have been shown to induce immunity to GAS [13], and the emm/enn fusion events alter the J14 sequence in every variant of the $\mathrm{M} 4^{\mathrm{C}}$ protein that we observed (Figure $2 \mathrm{~A}$ ). The enn gene has been reported to be transcribed at very low levels and may not be translated at all due to mutations early in the coding region [15]. Therefore, we speculate that previously circulating emm4 strains bearing the classic pattern E and canonical emm may not have engendered an immune response to the portion of the Enn protein now present in the $M 4^{\mathrm{C}}$ protein. Hence, these data suggest that the emm/enn gene fusion may have significant immunological effects on GAS-host interactions, but this needs to be experimentally tested.

In summary, we report the occurrence of a recombination event that has given rise to a chimeric emm gene and a novel emm pattern and provide evidence of its predominance among current emm4 strains. These data suggest that GAS mechanisms that alter the M protein are more varied than previously appreciated which could impact the efficacy of M protein-based vaccine strategies. 


\section{Acknowledgements}

We thank Dr. Bernie Beall for critical reading of the manuscript and suggestions regarding the origin of the different $\mathrm{M}$ protein variants.

\section{Financial Support}

This work was supported by National Institute of Allergy and Infectious Diseases (grant R21 AI132920) to S.A.S.

\section{Potential conflicts of interest}

The authors have no conflicts of interest to declare.

\section{Corresponding author}

Samuel A. Shelburne, MD, PhD, Department of Infectious Diseases, MD Anderson Cancer

Center, Unit 1460, 1515 Holcombe Blvd, Houston, TX, 77030, 713-792-3629 (o), 713-792-5381

(fax), sshelburne@mdanderson.org

Alternative corresponding author: Anthony R. Flores, MD, MPH, PhD, Division of Infectious

Diseases, Department of Pediatrics, and Center for Antimicrobial Resistance and Microbial

Genomics, UTHSC/McGovern Medical School, 6431 Fannin St., MSB 3.130, Houston, TX

77030, 713-500-6608 (o), 713-500-5688 (f), anthony.r.flores@uth.tmc.edu 


\section{References}

1. Sanderson-Smith, M., D.M. De Oliveira, J. Guglielmini, D.J. McMillan, T. Vu, J.K. Holien, A. Henningham, A.C. Steer, D.E. Bessen, J.B. Dale, N. Curtis, B.W. Beall, M.J. Walker, M.W. Parker, J.R. Carapetis, L. Van Melderen, K.S. Sriprakash, P.R. Smeesters, and M.P.S.G. The, A Systematic and Functional Classification of Streptococcus pyogenes That Serves as a New Tool for Molecular Typing and Vaccine Development. J Infect Dis, 2014.

2. Dale, J.B., M.R. Batzloff, P.P. Cleary, H.S. Courtney, M.F. Good, G. Grandi, S. Halperin, I.Y. Margarit, S. McNeil, M. Pandey, P.R. Smeesters, and A.C. Steer, Current Approaches to Group A Streptococcal Vaccine Development, in Streptococcus pyogenes : Basic Biology to Clinical Manifestations, J.J. Ferretti, D.L. Stevens, and V.A. Fischetti, Editors. 2016: Oklahoma City (OK).

3. Bessen, D.E., Molecular Basis of Serotyping and the Underlying Genetic Organization of Streptococcus pyogenes, in Streptococcus pyogenes : Basic Biology to Clinical Manifestations, J.J. Ferretti, D.L. Stevens, and V.A. Fischetti, Editors. 2016: Oklahoma City (OK).

4. Bessen, D.E., C.M. Sotir, T.L. Readdy, and S.K. Hollingshead, Genetic correlates of throat and skin isolates of group A streptococci. J Infect Dis, 1996. 173(4): p. 896-900.

5. Hollingshead, S.K., T.L. Readdy, D.L. Yung, and D.E. Bessen, Structural heterogeneity of the emm gene cluster in group A streptococci. Mol Microbiol, 1993. 8(4): p. 707-17. 
6. Beres, S.B. and J.M. Musser, Contribution of exogenous genetic elements to the group A Streptococcus metagenome. PLoS ONE, 2007. 2(8): p. e800.

7. Beres, S.B., G.L. Sylva, D.E. Sturdevant, C.N. Granville, M. Liu, S.M. Ricklefs, A.R. Whitney, L.D. Parkins, N.P. Hoe, G.J. Adams, D.E. Low, F.R. DeLeo, A. McGeer, and J.M. Musser, Genome-wide molecular dissection of serotype M3 group A Streptococcus strains causing two epidemics of invasive infections. Proc Natl Acad Sci U S A, 2004. 101(32): p. 11833-8.

8. Jones, K.F., S.K. Hollingshead, J.R. Scott, and V.A. Fischetti, Spontaneous M6 protein size mutants of group A streptococci display variation in antigenic and opsonogenic epitopes. Proc Natl Acad Sci U S A, 1988. 85(21): p. 8271-5.

9. Galloway-Pena, J., M.E. Clement, B.K. Sharma Kuinkel, F. Ruffin, A.R. Flores, H. Levinson, S.A. Shelburne, Z. Moore, and V.G. Fowler, Jr., Application of WholeGenome Sequencing to an Unusual Outbreak of Invasive Group A Streptococcal Disease. Open Forum Infect Dis, 2016. 3(1): p. ofw042.

10. Jacob, K.M., T. Spilker, J.J. LiPuma, S.R. Dawid, and M.E. Watson, Jr., Complete Genome Sequence of emm4 Streptococcus pyogenes MEW427, a Throat Isolate from a Child Meeting Clinical Criteria for Pediatric Autoimmune Neuropsychiatric Disorders Associated with Streptococcus (PANDAS). Genome Announc, 2016. 4(2).

11. Chochua, S., B.J. Metcalf, Z. Li, J. Rivers, S. Mathis, D. Jackson, R.E. Gertz, Jr., V. Srinivasan, R. Lynfield, C. Van Beneden, L. McGee, and B. Beall, Population and Whole Genome Sequence Based Characterization of Invasive Group A Streptococci Recovered in the United States during 2015. MBio, 2017. 8(5). 
12. Steer, A.C., I. Law, L. Matatolu, B.W. Beall, and J.R. Carapetis, Global emm type distribution of group A streptococci: systematic review and implications for vaccine development. Lancet Infect Dis, 2009. 9(10): p. 611-6.

13. Brandt, E.R., K.S. Sriprakash, R.I. Hobb, W.A. Hayman, W. Zeng, M.R. Batzloff, D.C. Jackson, and M.F. Good, New multi-determinant strategy for a group A streptococcal vaccine designed for the Australian Aboriginal population. Nat Med, 2000. 6(4): p. 455-9.

14. McMillan, D.J., P.A. Dreze, T. Vu, D.E. Bessen, J. Guglielmini, A.C. Steer, J.R. Carapetis, L. Van Melderen, K.S. Sriprakash, and P.R. Smeesters, Updated model of group A Streptococcus M proteins based on a comprehensive worldwide study. Clin Microbiol Infect, 2013. 19(5): p. E222-9.

15. Frost, H.R., M. Sanderson-Smith, M. Walker, A. Botteaux, and P.R. Smeesters, Group A streptococcal M-like proteins: From pathogenesis to vaccine potential. FEMS Microbiol Rev, 2018. 42(2): p. 193-204. 
A

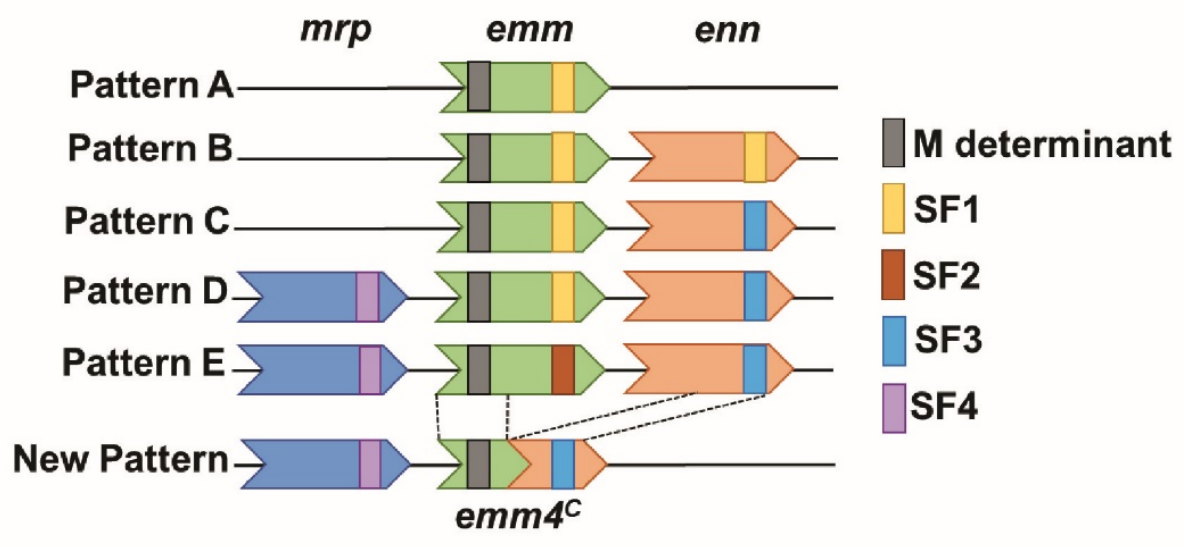

B

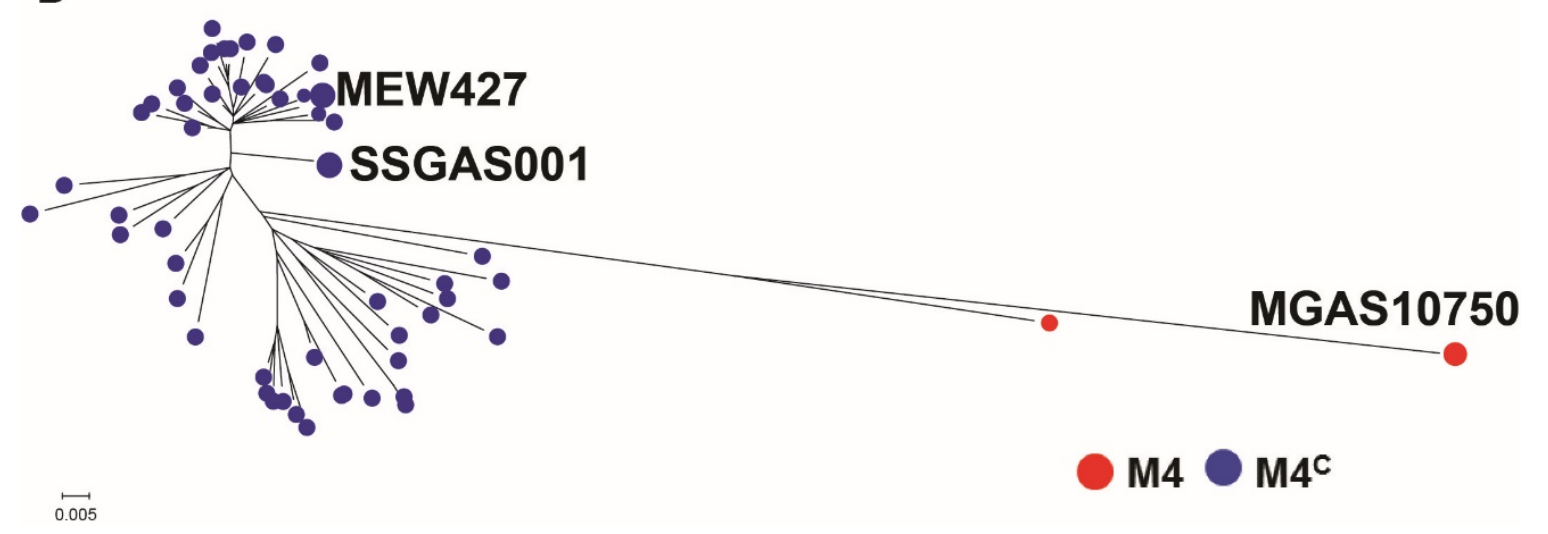

Figure 1. Characterization of GAS isolates. (A) Schematic diagram to compare the new emm pattern to existing patterns (adapted from reference [3]). The emm type-specific determinant and the subfamily of the PG domain is indicated. (B) Maximum-likelihood tree reconstructed on 814 core SNPs of emm4 GAS strains from CDC [11] along with publicly available emm4 GAS genomes (names are provided). The type of $\mathrm{M}$ protein is color coded as indicated in legend. 
A

MGAS10750 M

$1 \square$

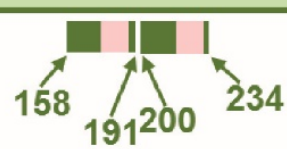

MGAS10750 Enn
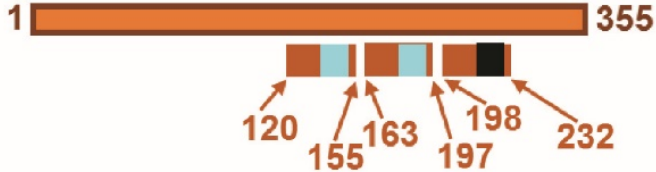

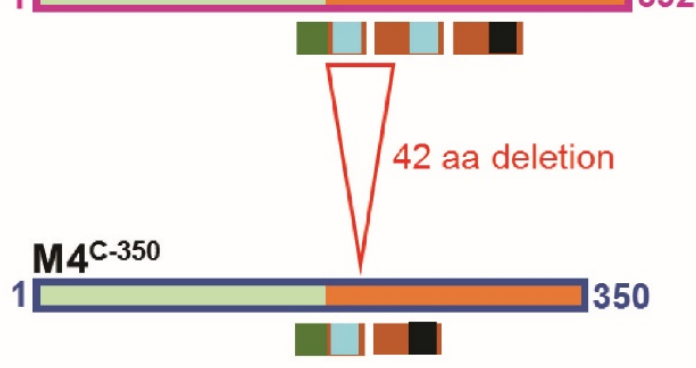

J14.2

J14.12

D J14.40

M CRR Enn CRR

B

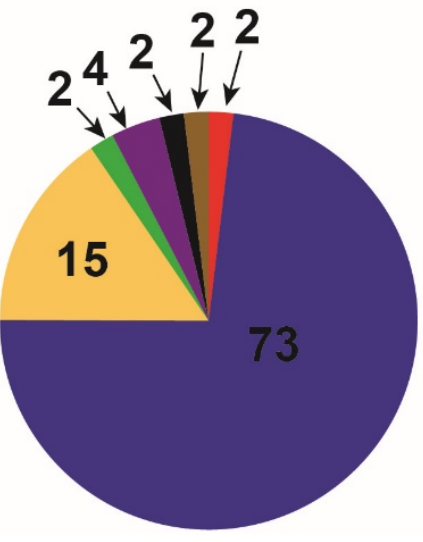

C

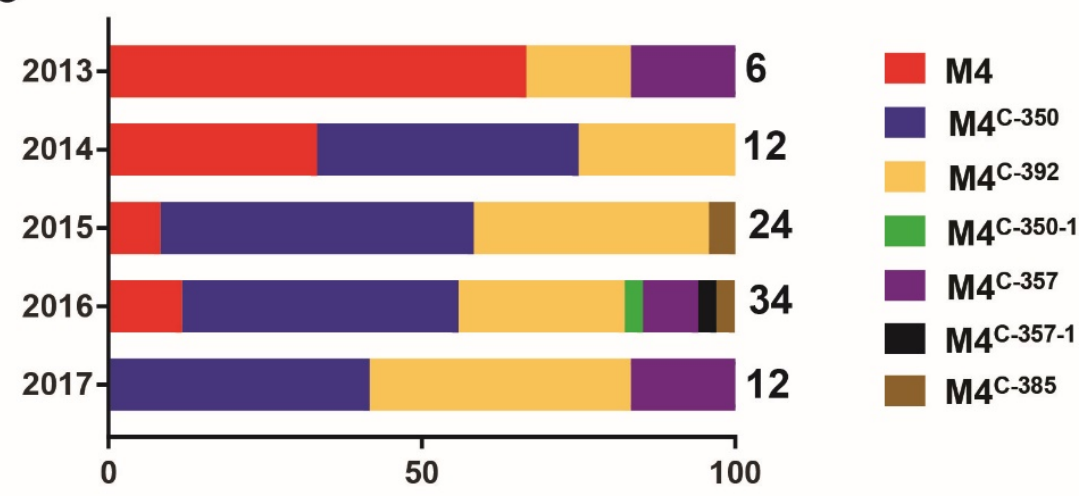

Figure 2. (A) Schematic diagram showing the two most common chimeric M protein variants.

The fusion point of the emm and enn genes is indicated by the change in box shading with numbers indicating the last amino acid from emm and first amino acid from enn respectively in $\mathrm{M} 4^{\mathrm{C}}$ protein. The 42 amino acid (126 nucleotide) deletion that is the difference between $\mathrm{M} 4^{\mathrm{C}-392}$ and $\mathrm{M} 4^{\mathrm{C}-350}$ is noted. C-terminal repeat regions (CRRs) along with J14 sequences are displayed as color-coded boxes. (B) Percent distribution of the canonical and chimeric M4 proteins in 52 emm4 GAS strains collected by the CDC in the United States in 2015 depicted as a pie-chart. Numbers indicate \% of total. (C) Distribution of the canonical M4 and the different $\mathrm{M} 4^{\mathrm{C}}$ variants 
identified in 88 emm4 strains isolated from patients at Texas Children's Hospital between 2013 and 2017 expressed as a percentage of the number of isolates per year. Total number of isolates in a given year is indicated on the right of the bars. There are relatively fewer isolates in 2013 because collection only began in May, 2013. For (B) and (C), the type of M protein is color coded as indicated in legend. 